\title{
Colaboradores de esta edición
}

Los siguientes científicos participaron en la elaboración de textos para la presente edición, en colaboración con los traductores y los miembros de la redacción.

Dra. Leonore Ballowitz

Profesora de Pediatría

Havelmatensteig 12

D-1000 Berlín 22

Dr. Hans-Jürgen Christen

Clínica Pediátrica de la Universidad

Georg-August

Robert-Koch-Str. 1

D-3400 Gotinga

Dra. Gisela Dallenbach-Hellweg

Profesora de Patologia

Instituto de Patología

A 2,2

D-6800 Mannheim

Dr. Gerhard Döring

Profesor de Ginecologia

Seeleite 9

D-8031 Seefeld 2

\section{Stefan Exner}

\section{Médico}

Schildhornstr. 72

D-1000 Berlin 41

Dra. Ingeborg Falck

Profesora de Medicina

Hospital Max Bürger de

Berlin-Charlottenburg

Sophie-Charlotten-Str. 115

D-1000 Berlín 19

Dr. Volker Faust

Profesor de Psiquiatría

Hospital Psiquiátrico Estatal Weissenau

Departamento de Psiquiatría I

de la Universidad de UIm

D-7980 Ravensburg-Weissenau

Dr. Dr. Erwin J. Haeberle

Profesor de Sexología

Instituto de Estudios Avanzados de

Sexualidad Humana y Universidad Estatal de

San Francisco

1100 Gough St

San Francisco, CA 94109, U.S.A.

Dr. Nikolaus Herden

Médico jefe del Departamento de Anestesio-

logía del Hospital General de Altona

Paul-Ehrlich-Str. 1

D-2000 Hamburgo 50
Dr. Joachim Hüter

Profesor de Ginecología

Médico jefe de la Clínica Ginecológica

del Hospital Municipal

Weinberg 1

D-3200 Hildesheim

Dr. Jürgen Kunze

Profesor de Pediatría

Clínica Pediátrica de la Universidad Libre

de Berlin

Instituto de Genética Humana

Kaiserin-Auguste-Viktoria-Haus

Heubnerweg 6

D-1000 Berlín 19

\section{Dr. Martin Lang}

Instituto de Medicamentos de la

Dirección General Federal de Salud Pública

Bundesgesundheitsamt

Seestr. 10

D-1000 Berlín 65

Dr. Rolf Meermann

Médico y Psicólogo, Director Clínico

del Hospital Psicosomático

Bombergallee 10

D-3280 Bad Pyrmont

Dr. Heinzpeter Moecke

Departamento de Anestesiología

del Hospital General de Altona

Paul-Ehrlich-Str. 1

D-2000 Hamburgo 50

Dr. Konrad Morgenroth

Profesor de Patología

Instituto de Patología

de la Universidad de Bochum

Universitätsstr. 15, Gebäude MA

D-4630 Bochum-Querenburg

Dr. Diether Neubert

Profesor de Farmacología

Instituto de Toxicología y Embriofarmacología de la Universidad Libre de Berlín

Garystr. 5

D-1000 Berlín 33

Dr. Detlef Petzoldt

Profesor de Dermatología

Hospital Dermatológico de la Universidad

Vosstr. 2

D-6900 Heidelberg 
Dr. Eckhardt Petri

Profesor de Ginecología

Hospital Municipal, Hospital de Formación

Académica de la Universidad de Maguncia

D-6580 Idar-Oberstein

Dr. Hans Peter Rosemeier

Profesor de Psicología

Instituto de Psicología Médica

de la Universidad Libre de Berlín

Habelschwerdter Allee 45

D-1000 Berlín 33

Dra. Hiltrudis Schütte

Hospital Albert Schweitzer

D-3410 Northeim

Dr. Jürgen Spona

Profesor de Endocrinología

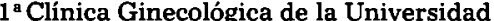

Spitalgasse 23

A-1090 Viena

Dr. Manfred Stauber

Profesor de Ginecología

Hospital Ginecológico de la

Universidad Libre de Berlín (Charlottenburg)

Pulsstr. 4-14

D-1000 Berlín 19
Dr. Johannes Staudt

Profesor de Anatomía

Instituto de Anatomía

de la Universidad Humboldt (Charité)

Philippstr. 12

DDR-1040 Berlín

Dr. Peter Voeltz

Departamento de Anestesiología

del Hospital General de Altona

Paul-Ehrlich-Str. 1

D-2000 Hamburgo 50

Dr. Dr. Helmut Weyers †

D-2160 Stade

Dr. Dr. Reinhard Wille

Profesor de Medicina Legal

Instituto de Investigación y Asesoramiento

Médico-Sexual de la Universidad

Hospitalstr. 17-19

D-2300 Kiel

Dra. Angela Zink

Socióloga, Directora del Departamento

de Epidemiología y Medicina Social

GSD $\mathrm{mbH}$

Stauffenbergstr. 13-14

D-1000 Berlín 30 